Historic, Archive Document

Do not assume content reflects current scientific knowledge, policies, or practices. 

Order Your Fall Garden and Field Seeds Tr--Don't Delay. WHOLESALE PRICE LIST

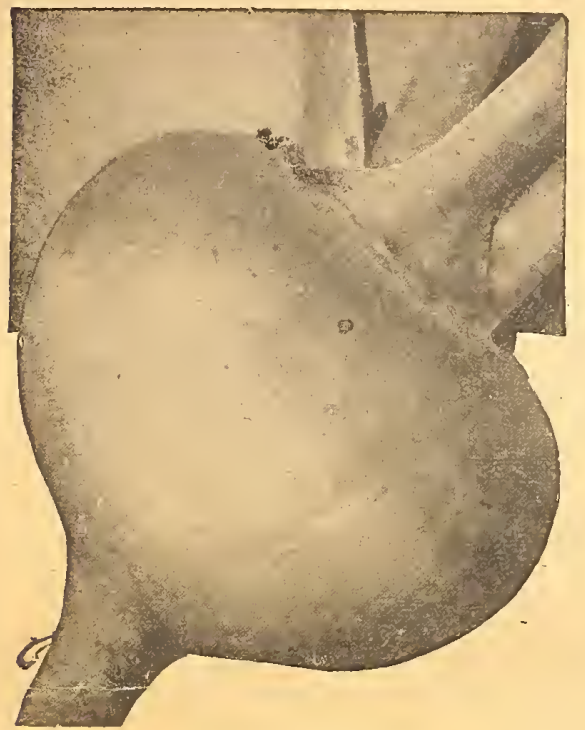

B. B. Kirkland Seed Co.

849 BROAD STREET, AUGUSTA, GA.

"Southern Seeds for Southern Soils"

Established 1910-17 Years of Seed Service.
I. INE EE

SEP 8 1927

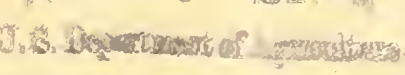

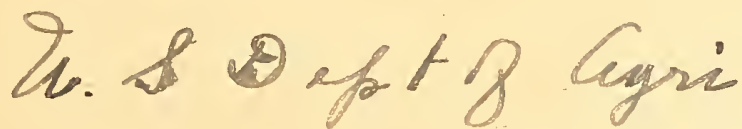

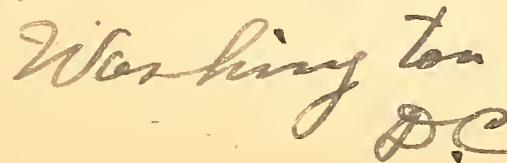

\section{WHOLESALE PRICE LIST of GARDEN SEEDS}

SELL KIRKLAND'S AMERICAN GROWN TURNIP SEEDS NOW. AII of our Turnip, Cabbage, Mustard Seeds are American Grown. A great many of our competitors offer European Grow
we think are inferior seed.

We are booking orders for Spring and early January shipment. Send us a list of your requirements and we will quote special prices.

\section{TURNIP} Purple Top White Globe......... $\begin{aligned} & \text { Lb. } \\ & 10 \text { or more } \\ & \$ 2.28\end{aligned}$ Purple Top Strap Leaf

Mammoth-Red Top Gloge

Long White Cowhorn....

White Egg

Golden Ball -.....................

Yellow or Amber Globe

Yellow or Am

Southern Prize

Extra Early White Milan

Extra Early P. T. Milan

Early Snowball

\section{CABBAGE}

Early Jersey Wàwefield........\$1.1 Charleston Wakefield ........... 1.1 All Head, Early

All Season

Carket

Surehead

Large Late Flat Dutch

Large Late Flat Dut

Chinese Pets

\section{CAULIFLOWER}

Earliest Snowball Per oz CARROTS

Half Long Danvers............ $\$ .50$ Guerande or Ox Heart........ .60 Chantenay, ....................... $\quad .70$ Large White Belgian.. ris cortur.

White or Cabbage Collard....\$ .45 True Georgia Collard............ . .45 North Carolina Short Stem.- $\quad .50$ Green Glaze .......................... .65

\section{CRESS}

10 Lbs.

Imp. American Purple Top...\$ $\$ 28$ \$.26 BEANS-DWARF, BUSH OR SNAP per lb. 50 lbs. 100 lbs Imp. Red Valentine........16 .15 Long Yellow Six Weeks.. .19

Extra Early Refugee...-... .17

Giant S. G. P.

Tences S.| G. P........... .16

Refugee or 1000 to $1 . . .16$

to $1 . . . . .18$

Imp. White Kidney........ 1

\section{BEANS-POLE OR RUNNINC}

Kentucky Wonder or lb. 50 lbs. $100 \mathrm{lbs}$ Old Homestead ...... $15 \quad .14 \quad .13$

White Seeded Kentucky

Wonder (................ 18

White Creaseback ........ $\quad .18 \quad .16 \quad .15$

Nancy Davis or Striped

Creaseback ................ .18 $.16 \quad .15$

BEANS_BUSH LIMA VARIETIES

Henderson or Der lb. $50 \mathrm{lbs} .100 \mathrm{lbs}$.

Sieva ......................... $\quad .15 \quad .15$ Burpee's Bush Lima......... . .18 $\quad .17 \quad .25$

BEANS-LIMA POLE or RUNNING

Carolina or Sieva Pole.. $1 \mathrm{lb} .100$ lbs. 500 lbs. Kirkland's Improved Pole

C

BEETS

Crimson Globe

Crosby's Egyptian

Detroit Dark Red

Imp. Early Blood Turnip

Early Eclipse

Long Smooth Blood

STOCK BEETS OR MANGELS

GoIden Tankard ..................\$ $.30 \quad$\begin{tabular}{ll} 
lb. & $10 \mathrm{lbs}$. \\
\hline & .25
\end{tabular} Long Red ................................ $.30 \quad \begin{aligned} & .25 \\ & .25\end{aligned}$

Curled
POP CORN

EGG PLANT

Florida High Bush............. 3.10

ENDIVE

\section{LETTUCE}

Black Seeded Simpson............ 1.30

Prize Head ...........................-1.40

Imp. Hanson

May King ...................-.-....... 1.40

New York or Wonderful...... 1.40

Crisp-as-Ice .......................... 1.40

Grand Rapid

Crand...- $\quad .90$

California Cream Butter......... 1.10

\section{MUSTARD}

Southern Giant Curled........ \$ . .35

Chinese Broad Leaved........
ONION

lb. $\quad 10 \mathrm{lbs}$

$.40 \quad .35$

Yellow Danvers .................... 1.50

Australian Brown ............... 1.00

Prizetaker …................. 1.50

Silver Skin or Portugal........ 1.65

Extra Early White Pearl......... 1.70

NASTURTIUM

Tall, Mixed

Dwarf, Mixed
Early Winningstadt

\section{SWEET PEAS}

$10 \mathrm{lbs}$.
$\$ 1.10$

1.10
1.40

2.00

1.10

1.90

1.10

1.05
1.10

1.10

1.05

Per lb.

$10 \mathrm{lbs}$.

$\$ .45$

.55
.65
.40

.40

$10 \mathrm{lbs}$.

$\$ .40$

.45

.60

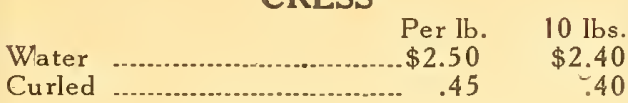

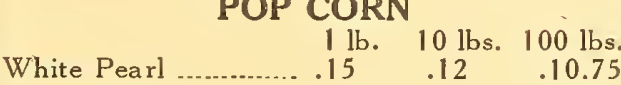

White Rice ……..........15 $\quad .12 \quad .15 \quad .12 \quad 9.75$

Queen's Colden .........15 $.15 \quad 13.00$

Per lb. $10 \mathrm{lbs}$

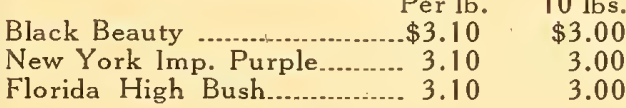

Grene Curled ..................... $\$ .75 \quad \$ \quad 10$ lbs

Moss Curled ..................... $80 \quad .75$

$10 \mathrm{lbs}$.

Big Boston ......................... $\$ 0$ or $\$ .85$

Red Wethersfield ..................\$1.50 $\begin{array}{lrr} & \text { Lb. } & 10 \mathrm{lbs} . \\ \text { Spencer Type, Mixed........... } \$ 1.25 & \$ 1.10 \\ \text { Choice Separate Colors....... } 1.75 & 1.60 \\ \text { Edkford Mixture Sweet Peas } & .90 & .80\end{array}$

GARDEN PEAS_SMOOTH SEED

All smooth Peas weigh 60 pounds per bushel. All wrinkled Peas 50 pounds per bushel.

Early Alaska

Ameer

White Marrowf

dible Pod

GARDEN PEAS-WRINKLED VARIETIES

Laxtonian ..................... Lb. 50 lbs. or more

Thomas Laxton

Premium Gem ...

Champion of England......18

PUIVIPRTIN

Firge Cheese or Kentucky \$.35 \$.30

Connecticut Field ............... .40

Cushaw or Crookneck

Green Striped Cushaw.......... .70

SUNFLOWER $100 \mathrm{lbs}$.
Lb. 50 lbs. or more

Mammoth Russian Sun- 15 .12

flower .......................

10 Lbs.

Early Scarlet Globe............. $\begin{aligned} & \text { Lb. } \\ & .45\end{aligned} \quad \begin{aligned} & \text { or more } \\ & \$ .40\end{aligned}$

$\begin{array}{rr}45 & .40\end{array}$

Long Scarlet Short Top.-.....- $.45 \quad .40$

White lcicle Scarlet.-........ $\quad .45$

White Tipped Scarlet........... .4

Sparkler White Tipped...-.-..... .4 45

French Breakfast ---

Long Black Spanish

Large White Spanish............

SPINACH

Bloomsdale Savoy ......... Lb. or mor

New Zealand

King of Denmark

Long Standing ..........................

SWISS CHARD

$10 \mathrm{Lbs}$

Lucullus

TOMATO

Spark's Earliana _..._........... $\$ 1.90$

Chalk's Early Jewel............... 1.90

John Barr

Gulf State Market................... 2.00

Acme

Matchless

Royal Red

Greater Baltin

Red Rock

Dwarf Sion

Dwarf Champio

Ponderosa

Beefsteak

Crimson Cushio

Redfield Beau

Yellow Plum

Norton Wilt Resistant.......... 5.00

Norduke Wilt Resistant...... 5.00 

B. B. Kirkland Seed Co. AUGUSTA, GEORGIA

Wholesale Price List for Merchants Only Long Distance Phone 298

"SOUTHERN SEEDS FOR SOUTHERN SOIL.S" ANNOUNCEMENT September 6th, 1927 We are pleased to announce to the trade that yre have purchased the business in
Augusta formerly operated by N. L. Willet Seed Company and more recently by
B Bolgiano Seed Company. We now have one of the best equipped seed houses in the South and are in position to give to the trade quicketr and more efficient service. Our stocks are all new and you will find KIRKLAND'S SEEDS TO GIVE YOUR MOST CRITCAL TRADE ENTIRE SATISFACTION. Our business has been built up for the pere you. Our location places us in position to give Quick Service reaching almost any
point in the Southern States in 24 to 48 hours. SEND US YOUR ORDERS. Prices quoted below are today's values, subject to market changes and goods being unsold, $f$. o. b. Augusta, Ga. We make no charge for packing or sacks except where seamless bags are
credit if desired.

\section{FIELD SEEDS} HAIRY VETCH 100-pound Lots .......................... $\$ 16.00$
220 -pound original bales per $100 \mathrm{lbs} .$.
15.50 NEW CROP CRIMSON CLOVER $\$ 20.50$ Per 100 pounds
220 pounds, per 100 pounds.................... 20.00 220 pounds, per
AUSTRIAN WINTER PEAS AUSTRIAN WINTER PEAS
Per 100 pounds ...................... 99.50
Lots of 500 pounds or more.............. 9.25

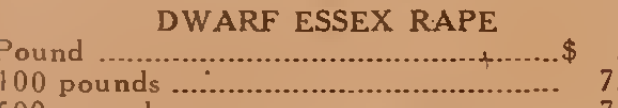

Pound
1000 pou
500 p SEED RYE-Genuine Abruzzi Bushel
10 -Bushel Lots

CAROLINA TALL GROWING RYE CAROLINA TALL GROWING RYE
Bushel 1.75
10 Bushel Lots 10.Bushel Lots
100 -Bushel Lots ROSEN RYE

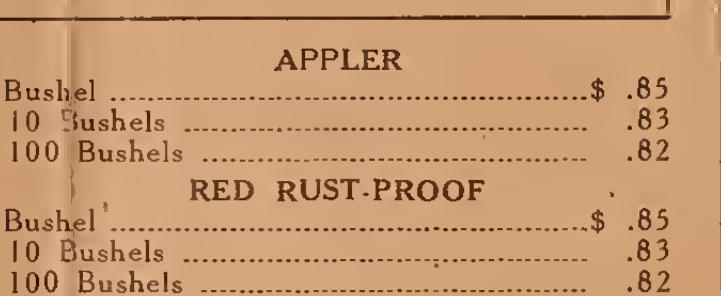

Bushel
10 -Bushel Lots SEED OATS-Fulghum Bushel
I0 Bushels.....
I00 Bushels

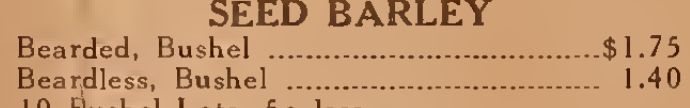

10 Eushel Lots, $5 \mathrm{c}$ less.

SEED WHEAT

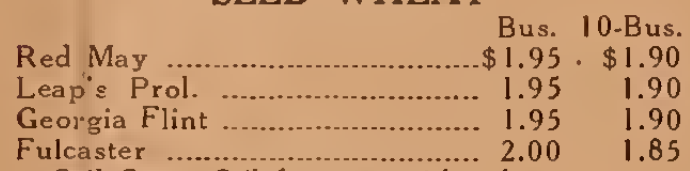

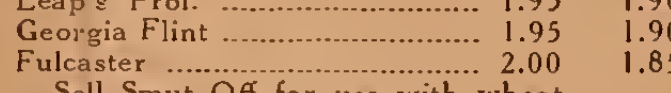
Sell Smut Of for use with wheat.

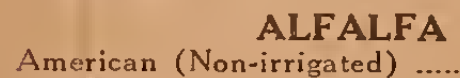

American (Non-
500 -lb. Lots

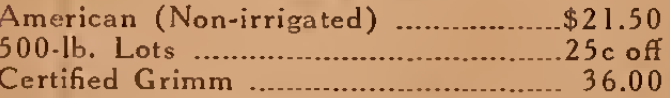

BURR CLOVER

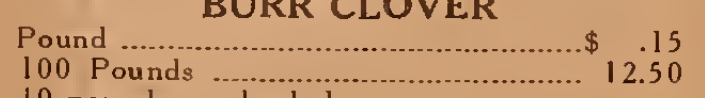

0 pounds per bushel.

Pound DALLAS GRASS

Pound
100 Pound
500 Pounds
.45
42.00

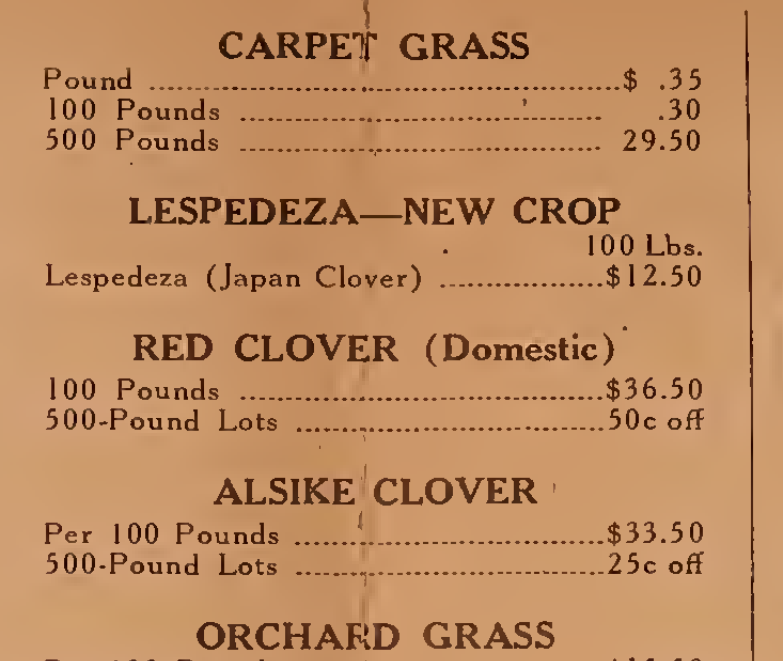

Per 100 Pounds .......................5 $\$ 15.50$
Lots of 500 Pounds or more......-...... 50 off

KENTUCKY BLUE GRASS

21 Pounds Seed, per 100 pounds........\$22.50

RED TOP OR HERD'S GRASS

Per 100 Pounds .................... \$22.75

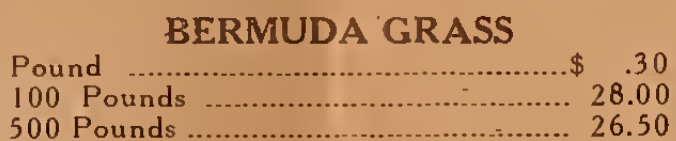

RYE GRASS

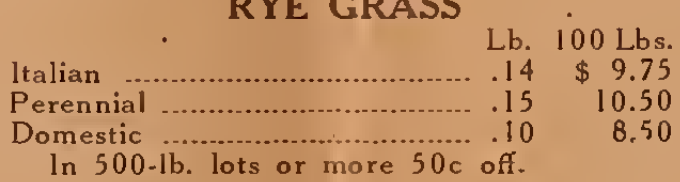
BAGS

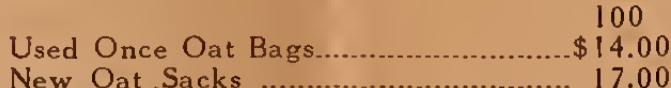

Jute Sacks (holds 2 $2 \frac{1}{2}$ bus. Corn, Peas,

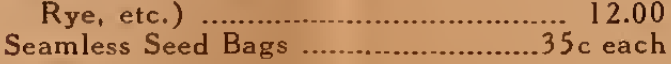

\section{Kirkland's Packet Seeds}

We are putting out this year our first seed
packet box. This box is sold outright to you and at the end of the seed selling season of this, issue New Seed the following year. which retail for $10 \mathrm{c}$ each; we sell you the most attractive and contain a full dime's worth

\section{Sell Kirkland's Lawn Grass Mixtures}

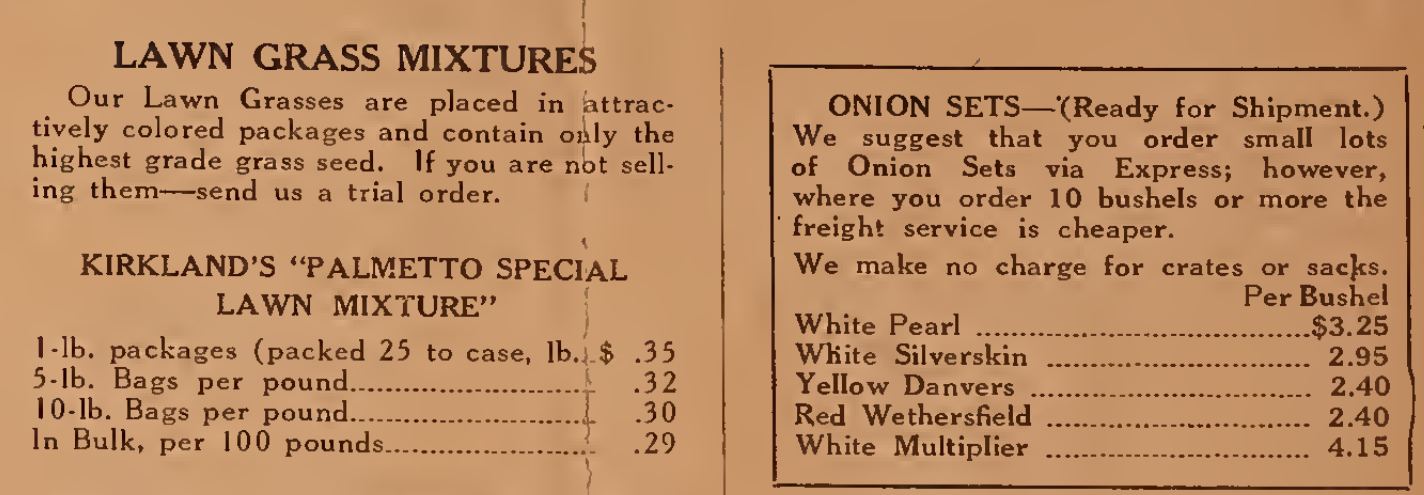

KIRKLAND'S “STAI-GREEN MIXTURE”

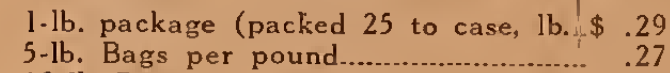

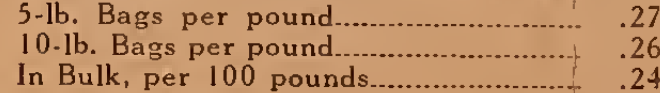

Per 100 Lbs.

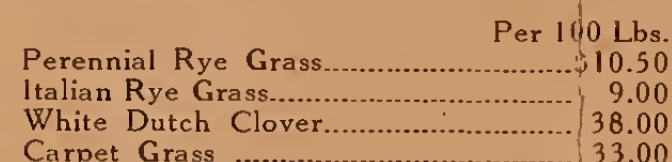

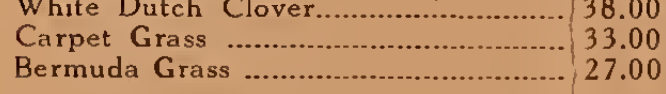

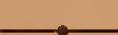

MISCELLANEOUS

Bulbs! Bulbs! We have a fine lot of Paperwhite Grand-
iflora Narcissus Bulbs READY FOR SHIP. MENT, $14 C M$ per $100 \$ 5.20$; per $\mathrm{M}$, . QUILS, ETC̣. Wizard Brand Sheep Manure, $100 \mathrm{ll}$. . sacks, 25 -lb. sacks, $30 \mathrm{c}$ per $100 \mathrm{lbs}$. more.

NITFOGEN INOCULATION - For Vetches,
Clovers, Soy Beans, Peas and Bean Alfalfa,

5. Bus. Size, Retail Price................... $\$ 4.75$

$\frac{1}{2}$-Bus. Size, Retail Price.......................... $\quad .60$

Bus. Size, Retail Price..........
Less $30 \%$ Discount Dealers.

$$
\longrightarrow
$$

BEE SUPPLIES-We are exclusive sales carload stock of these supplies at all t times.

ARDEN SEEDS - SHIPMENT MADE
Valencia Peanuts, $100 \mathrm{lbs} . . . . . . . . . . . . . . . . . \$ 8.50$ SAME DAY ORDER RECEIVED Don't miss sales on Garden Seeds-
Say what seed you need. We can to freight. When in hurry WIRE YOUR ORDERS AT OUR EXPENSE. Any items - not listed on this list, write for prices, or
send' us your orders for them. We will
price them rigbt.

\section{Poultry Feeds}

\section{Oyster Shell, $100 \mathrm{lbs}$.
Meat Scrap, $100 \mathrm{lbs} .$.}

Chicken Grit, 100
Charcoal, 50 lbs.

$60 \%$ Tankage, $100 \mathrm{lbs}$
Tobacco Dust, $100 \mathrm{lbs}$

Putnam Brooder Stoves, complete, each
4.75. Less $20 \%$ discount to dealer.

WE ARE BOOKING ORDERS FOR SPRING AND EARLY JANUARY SHIPMENT. SEND US A LIST OF YOUR REOUIREMENTS .AND WE WILL QUOTE SPECIAL PRICES. 
